\title{
Depth-sensing indentation applied to polymers: a comparison between standard methods of analysis in relation to the nature of the materials.
}

\author{
J Giró-Paloma ${ }^{1}$, J J Roa ${ }^{2,}$ *, A. M. Díez-Pascual ${ }^{3}$, E Rayón $^{4}$, A. Flores ${ }^{5}$, M Martínez $^{1}$, J M Chimenos ${ }^{1}$, A I. \\ Fernández ${ }^{1}$ \\ ${ }^{1}$ Universitat de Barcelona. Department of Materials Science and Metallurgical Engineering. Barcelona (Spain) \\ ${ }^{2}$ CIEFMA-Universitat Politècnica de Catalunya. Department de Ciència dels Materials i Eng. Metal·lúrgica. \\ Barcelona (Spain) \\ ${ }^{3}$ Institute of Polymer Science and Technology (ICTP-CSIC), Madrid (Spain) \\ ${ }^{4}$ Universidad Politécnica de Valencia. Materials Science Institute, Valencia (Spain) \\ ${ }^{5}$ Department of Macromolecular Physics, Institute for Structure of Matter (IEM-CSIC), Madrid, Spain. \\ * Corresponding author: joan.josep.roa@upc.edu
}

\begin{abstract}
Mechanical data (hardness and elastic modulus) from instrumented indentation testing are often extracted assuming linear elasticity in the initial portion of the unloading. The method is nowadays widely accepted as a convenient tool to interpret depth-sensing data, however it is a matter of controversy when applied to polymer materials due to their time-dependent behaviour. More recently, Loubet and co-workers applied continuous stiffness measurements (CSM), consisting of superimposing a small oscillation to the quasi-static component of loading, to the study of the mechanical properties of polymers and proposed a new model to account for the apparent increase in the contact area detected at the first stages of contact. The present work offers a comparative study between the Loubet's model using CSM and the procedure yielding a single reading from the onset of unloading. A wide range of thermoplastic polymer materials including glassy and semicrystalline polymers have been investigated. The most important equations employed for each method are summarized and the advantages and disadvantages of employing one procedure or the other are discussed. The differences found between the results obtained from both approaches are discussed in relation to the nature of the polymer material. A comparison between mechanical data extracted from indentation measurements and from classical dynamic mechanical analysis is offered.
\end{abstract}

Keywords: Nanoindentation; CSM; Hardness; Elastic modulus.

\section{Introduction}


Mechanical properties are a determinant criterion for the selection of a material for a specific application. Indentation testing represents a convenient way for the characterization of the mechanical properties. On the one hand, the major requirement for sample preparation is a suitable surface finish and this usually allows testing the specimen on its original assembly. On the other hand, the method can provide a wealth of mechanical data in a short period of time. In addition, the indentation technique offers information on the mechanical properties at a local scale and this is the main reason why it has gained increasing interest in the last decades. Coatings, thin films and nanocomposite materials are some examples where indentation testing is extremely useful [1-3]. Using depth-sensing indentation (DSI), the scale of deformation can approach the sub-micron or even the nanoscale. In DSI, the applied load is recorded as a function of penetration depth during a loading and an unloading cycle. The procedure most widely accepted to analyze the load-depth data from DSI to determine hardness and elastic modulus values was originally proposed by Doerner and Nix [4]. Doerner and Nix applied Sneddon's analysis of the contact between a punch and an elastic solid to the initial portion of the unloading curve. The procedure provided stiffness and contact area values opening up the possibility of deriving hardness and elastic modulus from depth-sensing data. Oliver and Pharr further proposed an analysis procedure to account for nonlinear initial unloadings present when indenters other than flat cylindrical punches are employed [5].

Nanoindentation has been proved to be well suited for the characterization of small volumes of ceramics or metals, such as single grains or phases in a composite [6], dislocation dynamics [7], small structures [8] or thin films and coatings [9]. Oliver and Pharres analysis has been successfully applied to these materials providing reliable values of hardness and elastic modulus [5]. However, the application of indentation testing to polymers is still behind that of metals and ceramics, especially with respect to depth-sensing devices, although some efforts have been done in the area [10-12]. This is due to the time dependent properties of polymers that question the validity of applying Sneddon "s analysis to the onset of unloading [10-12]. In addition, polymer materials demand a method that can provide information on the viscoelastic and viscoplastic properties and not simply the values of hardness and elastic modulus. A step forward was achieved when commercial DSI devices introduced the possibility of applying an oscillating force to the force signal $[\mathbf{5}, \mathbf{1 3}]$. The method is commonly known as continuous stiffness measurement (CSM) or dynamic DSI and opened up the possibility of using DSI devices as sub-micro or nano-scale dynamic mechanical analyzers. Loubet et al. were the first to successfully apply CSM to polymers suggesting a 
route for the derivation of hardness and elastic modulus in dynamic conditions [14]. In addition, these authors developed a model that introduced the concept on an ,apparent ${ }^{\text {ee }}$ tip defect, $h_{o}$, accounting for a shift in the contact point detected for polymer materials. In the model, the $h_{o}$ value is added to the plastic depth to estimate the ,apparente contact area. In turn, such procedure yields mechanical data independent of the indentation depth.

Several effects should be taken into account when applying DSI to ,soft ${ }^{\text {te }}$ materials such as polymers: (i) the variation of the mechanical properties with the strain-rate and with the frequency of the modulated load, (ii) the interaction forces between the indenter and the sample (e.g. forces of Van der Waals, repulsive or attractive forces, and others), and (iii) the conditions of testing that should be optimized to achieve a high sensitivity to stiffness variations in order to detect the surface efficiently. Concerning point (iii) above, the application of an oscillating force usually improves the surface detection; however, for polymers with marked elastomeric character large errors are still introduced in the surface detection that lead to uncertainties in the determination of the mechanical properties [15].

The main aim of this study is the application of Loubetes and Oliver and Pharres analysis to a wide range of thermoplastic materials including glassy and semicrystalline polymers. A comparison between the hardness and elastic modulus values determined using either method will be carried out. Moreover, a discussion on the main advantages/disadvantages of each method will be offered. In addition, the validity of each model is discussed in relation to the nature of the polymer material. Dynamic mechanical analysis (DMA) will be employed to achieve modulus values for comparison with those extracted from the indentation analysis. We intend to provide some bases to decide a priori which indentation method would be most appropriate for a specific polymer material.

\section{Experimental procedure}

\subsection{Samples}

A commercial set of polymeric samples supplied by SIDILAB, S.L. was used. The selected polymers are: polyamide/Nylon 6 (PA6), poly(methyl methacrylate) (PMMA), polycarbonate (PC), amorphous polyethylene terephthalate (PET), acrylonitrile butadiene styrene (ABS), polypropylene (PP) and polystyrene (PS). Samples of 75x11x2 mm were cut transversally obtaining specimens of $20 \times 11 \times 2 \mathrm{~mm}$ size. The different samples were directly glued onto a metallic holder to perform the nanoindentation tests. 


\subsection{Depth-sensing instrumentation}

Nanoindentation tests were performed with a Nanoindenter G200 (Agilent Technologies, Chandler, AZ) provided with Continuous Stiffness Measurement (CSM). A Berkovich diamond tip was employed. Tip shape calibration was performed following the procedure described in detail in $[\mathbf{5 , 1 3}$. Measurements were also corrected for the machine compliance. Experiments were carried out at room temperature $\left(20{ }^{\circ} \mathrm{C}\right)$. The indentation tests were performed using the following sequence: the load was incremented at a constant strain rate of $0.2 \mathrm{~s}^{-1}$ up to a peak load of $500 \mathrm{mN}$. During this loading cycle, a small oscillating force of 2 $\mathrm{nm}$ of amplitude and $45 \mathrm{~Hz}$ of frequency was superimposed. This period was followed by a peak hold time of $10 \mathrm{~s}$ after which the load was withdrawn up to $90 \%$ of the peak load. Finally, a reloading/fully unloading period is carried out.

The Loubet (see Annex A) and the Oliver and Pharr (see Annex B) methods were employed to extract the mechanical properties using the Test Works 4 Professional level software. The Loubet method uses the CSM option providing a continuous measure of the indentation stiffness during the loading cycle. In this way, values of the reduced modulus and the indentation hardness are obtained as a function of the indentation depth. In contrast, these mechanical data in the Oliver and Pharr approach are obtained from the analysis of the final unloading curve.

A homogeneous array of sixteen indentation imprints was performed on each sample. Each imprint was separated a constant distance (around 25 times the maximum indentation depth) in order to avoid the interaction of the plastic deformation field between indentations. The residual imprints were directly visualized by the optical microscopy coupled to the G200.

\subsection{Dynamic mechanical analysis}

A dynamic mechanical analyzer (Mettler DMA 861) was used to measure the storage modulus $E^{\prime}$ and $\tan \delta$ of the materials. Experiments were carried out in the tensile mode by heating from -100 to $200{ }^{\circ} \mathrm{C}$ with a ramping rate of $2{ }^{\circ} \mathrm{C} / \mathrm{min}$ at a frequency of $1 \mathrm{~Hz}$. A dynamic force of $6 \mathrm{~N}$ was used oscillating at fixed frequency and amplitude of $30 \mu \mathrm{m}$. Figure 1 shows the dynamic mechanical spectra for the different samples. The peaks in $\tan \delta$ curves are related to transitions in polymers. The peak at higher temperature is the $\alpha$-transition or glass transition, and covers a range of temperatures, of which the so-called glass transition 
temperature $\left(T_{g}\right)$ is an indicative value determined based on an agreed criterion [16]. In this work, $T_{g}$ values were determined from the peak maximum of $\tan \delta$ vs. temperature curves [17]. Lower temperature peaks correspond to secondary transitions $(\beta$ and $\gamma)$ that are thought to be related to local small-scale chain motions. In case of the ABS terpolymer, two $\mathrm{T}_{\mathrm{g} S}$ are observed: one associated with the poly(butadiene) at $-65^{\circ} \mathrm{C}$ with very small $\tan \delta$ magnitude and other at $\approx 110^{\circ} \mathrm{C}$ ascribed to the poly(styrene-co-acrylonitrile) segments. Table 1 collects the $E^{\prime}$ value for each polymer sample determined at room temperature together with the corresponding $T_{g}$ value.

\section{Results and discussion}

\subsection{Imprints visualization}

The residual area after indentation is different for each sample and, taking into account that experiments were carried out using the same maximum load, yields qualitative information about the hardness of each material. Figure 2 shows, as an example, the residual imprints performed on the surface of two polymer glasses (PC and PMMA). The scratches and cracks at the sample surfaces, especially evident in the case of PMMA, existed before the indentation measurements and were found not to alter the results at high penetration depths (above $5 \mu \mathrm{m}$ ). A quick inspection of the residual impressions reveals that the hardness of PC is smaller than that of PMMA. Indeed, analysis of the indentation depth data by either method (Oliver and Pharr or Loubet) confirms this finding (see results below).

\subsection{Considerations: Loubet method}

\subsubsection{Tip defect}

A typical representation of the Harmonic Contact Stiffness, HCS, vs. the plastic deformation depth, $h_{r}$, is shown in Figure 3. The tip defect $\left(h_{o}\right)$ can be calculated from the $\mathrm{x}$-axis intercept following [13]:

$H C S=b\left(h_{r}{ }^{\prime}+h_{0}\right)$ 
where $b$ is the slope of the straight line. The different tip defect $\left(h_{o}\right)$ determined for each sample can be summarized in Table 2. The existence of a linear relationship between these parameters, over a critical plastic depth, is a proof that these polymeric materials meet the constant $E$ assumption.

Table 2 shows significant variations in the $h_{o}$ values of the different samples with respect to each other. This could be related to a different adhesion of the sample to the tip that in turn, should be a consequence of the material characteristics. One would expect that samples with the highest viscous character, i.e., those with $T_{g}$ below room temperature and a significant amount of amorphous phase, would exhibit the highest adhesion to the tip (largest $h_{o}$ values). Indeed, this would explain why PP exhibits one of the highest $h_{o}$ values $\left(T_{g}\right.$ of $\mathrm{PP} \approx 10{ }^{\circ} \mathrm{C}$; degree of crystallinity $\approx 0.4-0.7[10,18])$ It would also explain the large difference found between the $h_{o}$ values of PP and that of glassy polymers such as PC, amorphous PET and PMMA, all of them with $T_{g}$ well above room temperature. In case of the ABS material, the butadiene blocks have already experienced the glass transition at room temperature and hence, this could impart enhanced tip-sample interaction (higher $h_{o}$ value) with respect to the rest of glassy polymers. However, the data in table 2 suggest that additional factors other than the viscous character of the polymer material are influencing the apparent tip defect values. Indeed, PS exhibits a significantly higher $h_{o}$ value that other glassy materials (PC, amorphous PET, PMMA). Moreover, PA6 $\left(T_{g} \approx 50{ }^{\circ} \mathrm{C}\right.$; degree of crystallinity $\left.\approx 0.3-0.4,[19]\right)$ displays an $h_{o}$ value very close to that of PP although the glass transition temperature of the former material is shifted by about $40{ }^{\circ} \mathrm{C}$ with respect to that of the latter one. Hence, we must conclude that other factors such as the surface roughness could also play an important role in the tip-sample interaction.

\subsubsection{Loading/Unloading curve}

Using the $h_{o}$ values of Table 2, corrected $P$ - $h$ curves are obtained and subsequently used to recalculate the indentation hardness $\left(H_{I T}\right)$ and elastic modulus $(E)$ following the procedure described in Annex A. In practice, these corrections are easily introduced using the Testworks 4 software. As an example, Figure 4 shows a typical loading-unloading-reloading curve after correction of the tip defect. It is noteworthy the overlapping of the unloading-reloading cycles.

\subsection{Considerations: Single reading from the unloading}


In the Oliver and Pharr analysis, $H_{I T}$ and $E$ are straightforward determined from the initial portion of the final unloading curve. One single reading at the end of the test is extracted instead of a continuous measurement of the mechanical properties as provided by the CSM option. In this case, the $P$ - $h$ curves are only corrected for the point of initial contact and not for the ,apparente tip defect. This can be easily done examining the evolution of the contact stiffness with time. The method is less time-consuming but does not take into account the time-dependent properties of polymers.

In the following section we will compare and discuss the mechanical properties obtained using both, Oliver and Pharr and Loubet's methods.

\subsection{Mechanical properties}

Figures 5a and $\mathbf{b}$ show the evolution of $H_{I T}$ and $E$, respectively, against the penetration depth for each polymer using the Loubet method. It is found that for all materials, $H_{I T}$ and $E$ reach a plateau at $\approx 5 \mu \mathrm{m}$. The indentation behaviour at small penetration depths should be a consequence of superficial defects and surface roughness. However, Figure 5 shows that, for some of the materials such as PET, PC or PMMA, the mechanical data seem to systematically decrease with increasing penetration depth up to the plateau value. Because the experiments were carried out in constant strain rate conditions and a careful correction for the apparent tip defect has been carried out, we must conclude that this result is due to a surface hardening effect. Preceding indentation work on PC, PS and PMMA, reported enhanced hardness and modulus values at the surface layer with respect to the bulk [20]. The authors explained this result as a modification of the material properties at the surface due to exposure to light and air, or due to the fabrication process itself. Similar ageing, oxidation effects or other morphological gradients across the samples arising from the manufacturing process could take place in our materials.

Let us know focus on the plateau $H_{I T}$ and $E$ 'bulk' values shown in Figures $5 \mathbf{a}$ and $\mathbf{5 b}$. Two different range of values are observed: $i)$ high values for: PMMA > PC > PET $\left(H_{I T}>200\right.$ $\mathrm{MPa}, E>3 \mathrm{GPa})$, and ii) low values for: PP, PA, ABS and PS ( $\left.H_{I T}<200 \mathrm{MPa}, E<3 \mathrm{GPa}\right)$. It is noteworthy that the modulus values represented in Figure $\mathbf{5 b}$ are in good agreement with the $E^{\prime}$ data obtained from DMA tests (see table 1). In both experiments, PMMA exhibits the highest modulus value, followed by glassy PET and PC, which display values close to each other. Both, indentation and DMA studies reveal significant lower modulus values for the rest 
of polymer materials (PP, PA, ABS, PS). Comparison between the modulus values obtained by means of indentation and those from DMA reveal differences $\leq 20 \%$ except for PS where the modulus value obtained using the Loubet method is about $40 \%$ higher than $\mathrm{E}^{\prime}$.

Table 3 summarizes the mechanical properties determined using Loubet and Oliver and Pharr methods. It is clearly seen that the $H_{I T}$ and $E$ values are lower when the Loubet method is used. Two main reasons can explain this result: $i$ ) dynamic testing was used when applying the Loubet method; here, a viscoelastic model is implicit in the derivation of the harmonic stiffness that, in turn, is employed to determine $H_{I T}$ and $E$; and $i i$ ) the contact depth (and hence, the contact area) is larger in case of Loubet ${ }^{\text {ee }} \mathrm{s}$ method due to the contribution of an „apparent" tip effect. Although the elastic modulus values obtained using either method are consistent with the storage modulus values obtained from DMA tests, it seems that Loubet method tend to yield modulus values closer to those derived from DMA.

Figure 6 plots the mechanical properties obtained using the Loubet method versus the Oliver and Pharr analysis. A straight line representing the identity function is included on each plot. It is clearly seen that Loubete's method tends to yield smaller values of $H_{I T}$ and $E$ than Oliver and Pharr, as argued above. However, most interesting is the fact that some materials deviate to a larger extent than others. This prompted us to correlate such deviations with the corresponding glass transition temperatures obtained from DMA (see table 1). Figures 7a and b shows the plot of $H_{\text {Loubet }} / H_{\text {Oliver\&Pharr }}$ and $E_{\text {Loubet }} / E_{\text {Oliver\&Pharr }}$, respectively, as a function of the difference between the glass transition of each material and the temperature of measurement $\left(T_{g}-20{ }^{\circ} \mathrm{C}\right)$. In case of the ABS terpolymer, we have adopted the highest $T_{g}$ value $\left(\approx 110^{\circ} \mathrm{C}\right)$ because the integrated area of the corresponding $\tan \delta$ transition is substantially larger than that of the low- $T_{g} \alpha$-transition (see Figure 1b). It is seen that the elastic modulus ratio tend to increase with increasing glass transition values. This observation is most interesting because it suggests that the validity of the Oliver and Pharr approach is restricted to polymers with a low viscous character, i.e. with $T_{g}$ well above the temperature of measurement. Hence, for room temperature indentation measurements, Loubet ${ }^{\text {te }}$ method should be preferentially applied if the polymer material exhibits a $T_{g}$ below or on the vicinity of room temperature. On the other hand, Figure $\mathbf{7 b}$ shows that a clear trend with $T_{g}$ is not observed for the hardness ratio. The viscous character of a polymer material clearly influences the material ${ }^{\text {ee }} \mathrm{s}$ behavior upon unloading that in turn determines the stiffness values calculated at peak load using Oliver and Pharr analysis ( $S_{\text {contact }}$ in equation B1). Because the $E$ values are directly related to $S_{\text {contact, }}$ the selection of a method that adequately contemplates viscoelasticity such as Loubet ${ }^{e e} \mathrm{~s}$ is of great importance in the case of polymers with a marked 
viscous character. However, in the case of $H_{I T}$, no clear recommendation to use one method or the other is inferred from our results because this magnitude is not directly related to the stiffness. Hence, in this case, either method seems to be equally valid for the derivation of hardness values.

\section{Conclusions}

The most common methods employed for analysis of the mechanical properties of polymers, Oliver and Pharres s analysis and Loubet's, have been critically examined.

In case of application of Loubetes method, polymer materials with a marked viscous character (glass transition values below room temperature) exhibit large values of the apparent tip defect. In addition, this parameter is also envisaged to be influenced by surface roughness.

Comparison of the hardness and elastic modulus values derived from both approaches suggests that Loubet's method tend to yield lower values of the mechanical properties, closer to the modulus data determined by means of DMA. This effect is attributed to two main factors: i) Loubet "s model uses a dynamic approach to extract stiffness measurements that, in turn, are employed to derive $H_{I T}$ and $E$; ii) the contact depth is larger in case of Loubet $\mathrm{s}$ method due to the contribution of the ,apparent" tip effect.

Results obtained from the analysis of the onset of unloading suggest that this method should be limited to polymer materials with a low viscous character, i.e., with $T_{g}$ well above the temperature of measurement.

\section{Acknowledgements}

The authors would like to thank C. Ferrer and M. D. Salvador of the Universidad Politécnica de Valencia, the European Union (COST Action TU0802), and the Catalan Government for the quality accreditation given to research group DIOPMA (2009 SGR 645). One of us (AF) thanks the MICINN (Ministerio de Ciencia e Innovación), Spain, for financial support under the grant FIS2010-18069. We would like to thank the Linguistic Services at the Universitat de Barcelona for linguistic and stylistic advice.

\section{Annex A: Loubet method [14]}


In this method, the contact depth is obtained using the following equation:

$h_{c}=\alpha\left(h_{r}^{\prime}+h_{0}\right)$

where $h_{o}$ represents the apparent tip defect, i.e., the apparent missing portion of the diamond from the height of a perfect Berkovich pyramid. The $h_{o}$ parameter does not represent a "real" tip defect but is introduced in Loubet"s model as an "apparent" value that is necessary to ensure a constant stiffness value through the thickness of the material. Loubet et al. related the $h_{o}$ parameter to specific phenomena in polymer materials (tip-sample interaction, incorrect detection of the sample surface, etc). The contact depth in Loubet's model, $h_{c}$, is determined by adding the plastic depth, $h_{r}$ ', to the apparent tip defect. The $\alpha$ parameter is a coefficient, which is equal to 1.2 when a Berkovich tip indenter is employed.

The contact area $\left(A_{c}\right)$ can be calculated using the following expression:

$$
A_{c}=\beta \alpha^{2}\left(h_{r}^{\prime}+h_{0}\right)^{2}
$$

where $\beta$ is a shape factor, which for a Berkovich diamond tip indenter is equal to 24.56 . The indentation hardness $\left(H_{I T}\right)$ value can be defined as:

$H_{\mathrm{IT}}=\frac{P_{\max }}{A}=\frac{P_{\text {max }}}{\beta \alpha^{2}(h+h)_{0}^{2}}$

where $P_{\max }$ is the maximum applied load during the nanoindentation test. The effective elastic modulus $\left(E_{\text {eff }}\right)$ can be obtained as:

$$
E_{\text {eff }}=\frac{1}{\beta} \frac{\sqrt{\pi b}}{2 \alpha b}
$$

where the $b$ parameter is defined in equation 1 . The elastic modulus can be extracted using:

$$
\frac{1}{E_{e f f}}=\frac{1-v^{2}}{\square_{i}} \vec{E}_{i} \quad \frac{1-v^{2}}{E}
$$


where $v$ is Poisson ${ }^{\text {ee }}$ ratio for the material. The subindex $i$ denotes the parameters for the diamond tip indenter (0.07 and $1141 \mathrm{GPa}$, respectively [5]).

\section{Annex B: Oliver and Pharr analysis [4,5]}

The contact depth using this method can be obtained following:

$$
h_{c}=h_{\max }-\varepsilon \frac{P_{\max }}{S_{\text {contact }}}
$$

where $h_{\max }$ is the maximum indentation depth, $\varepsilon$ is a constant $(0.75$ for a Berkovich indenter), $P_{\max }$ is the maximum indentation load and $S_{\text {contact }}$ is the contact stiffness calculated from the unloading curve.

The contact area $\left(A_{c}\right)$ is calculated using the following expression:

$$
A_{c}=24.56 h^{2}
$$

$H_{I T}$ can be estimated using equation A 3 with $h_{o}=0$, and $E_{\text {eff }}$ can be obtained as follows:

$$
E_{\text {eff }}=\frac{\sqrt{ } \pi}{2} \frac{S_{\text {contact }}}{\sqrt{A}{ }_{c}}
$$

Finally, equation A5 can be used to derive the elastic modulus of the material.

\section{References}

[1] Bull SJ. J Phys D Appl Phys 2005;38:R393-R413.

[2] Hay J, Crawford B. J Mater Res 2011;26(6):727-38.

[3] Liu TX, Phang IY, Shen L, Chow SY, Zhang WD. Macromolecules 2004;37:7214-22.

[4] Doerner MF, Nix WD. J Mater Res 1986; 1(4):601-9.

[5] Oliver WC, Pharr GM. J Mater Res 1992;7:1564.

[6] Roa JJ, Capdevila XG, Martínez M, Espiell F, Segarra M. Nanotechnology 2007;18:1-6.

[7] Gaillard Y, Tromas C, Woirgard J. Acta Mater 2006;54:1409-17. 
[8] Choi Y, Suresh S. Scripta Mater 2003;48:249-54.

[9] Roa JJ, Gilioli E, Bissoli F, Patini F, Rampino S, Capdevila XG, Segarra M. Thin Solid Films 2009;518:227-32.

[10] Flores A, Ania F, Balta-Calleja FJ. Polymer 2009;50:729-46.

[11] VanLandingham MR, Villarrubia JS, Guthrie WF, Meyers GF. Macromol Symp 2001;167:15-43.

[12] Kranenburg JM, Tweedie CA, Van Vliet KJ, Schubert US. Adv Mater 2009;21:3551-61.

[13] Oliver WC, Pharr GM. J Mater Res 2004;19:3-20.

[14] Hochstetter G, Jimenez A, Loubet JL. J Macromol Sci Phys B38 1999;5\&6:681-92.

[15] Deuschle J, Enders S, Arzt E. J Mater Res 2007;22:3107-19.

[16] Kalogeras IM, Hagg Lobland HE. J. Mater. Ed 2012; 34:69-94.

[17] Menard KP. Thermal transitions and their Measurement. In: Witold Brostow, editor. Performance of Plastics. Carl Hanser Verlag: München 2001. pp.163-171).

[18] Meille SV, Brückner S. X-ray Scattering. In: Karger Kocsis J, editor. Polypropylene. An A-Z reference. Kluwer Academic Publishers; Dordrecht 1999. p. 891.

[19] Brucato V, Piccarolo S, Titomanlio G. Macromol Chem Macromol Symp 1993, 68:24555.

[20] Briscoe BJ, Fiori L, Pelillo E. J Phys D: Appl Phys 1998, 31:2395-405. 


\section{Table captions}

Table 1. Storage modulus $\left(E^{\prime}\right)$ at $25^{\circ} \mathrm{C}$ and glass transition temperature $\left(T_{g}\right)$ values obtained from DMA measurements.

Table 2. Tip defect $\left(h_{o}\right)$ at maximum applied load for each polymer studied.

Table 3. Summary of the mechanical properties for each polymer studied determined using the Loubet and Oliver\&Pharr methods at maximum indentation depth. 
Table 1

Table 1

\begin{tabular}{|c|c|c|}
\hline Material & $\boldsymbol{E}^{\prime}(\mathbf{G P a})$ & $\boldsymbol{T}_{\boldsymbol{g}}\left({ }^{\mathbf{}} \mathbf{C}\right)$ \\
\hline $\boldsymbol{P S}$ & 1.3 & 92 \\
\hline $\boldsymbol{A B S}$ & 1.7 & $-65,109^{\mathrm{a}}$ \\
\hline $\boldsymbol{P A}$ & 1.8 & 51 \\
\hline $\boldsymbol{P C}$ & 2.9 & 145 \\
\hline $\boldsymbol{P M M A}$ & 4.0 & 107 \\
\hline $\boldsymbol{P E T}$ & 2.7 & 71 \\
\hline $\boldsymbol{P P}$ & 1.6 & 8 \\
\hline
\end{tabular}

${ }^{\text {a }}$ The two $T_{g}$ s correspond to the poly(butadiene) and poly(styrene-co-acrylonitrile) segments, respectively. 
Table 2

Table 2

\begin{tabular}{lccccccc}
\hline Material & PS & PA & PC & PET & PP & PMMA & ABS \\
\hline$h_{o}(\mathrm{~nm})$ & 76 & 82 & 22 & 11 & 85 & 5 & 86 \\
\hline
\end{tabular}




\section{Table 3}

\begin{tabular}{|l|l|l|l|l|}
\hline \multirow{2}{*}{ Material } & \multicolumn{2}{|l|}{ Loubet method } & \multicolumn{2}{l|}{ Oliver \& Pharr method } \\
\cline { 2 - 5 } & $\boldsymbol{H}(\mathrm{MPa})$ & $\boldsymbol{E}(\mathrm{GPa})$ & $\mathrm{H}(\mathrm{MPa})$ & $\boldsymbol{E}(\mathrm{GPa})$ \\
\hline$A B S$ & $118 \pm 9$ & $2.1 \pm 0.1$ & $135.2 \pm 0.4$ & $2.7 \pm 0.4$ \\
\hline$P C$ & $218 \pm 3$ & $3.0 \pm 0.1$ & $236.1 \pm 0.3$ & $3.6 \pm 0.3$ \\
\hline$P M M A$ & $317 \pm 16$ & $4.9 \pm 0.1$ & $344.3 \pm 0.7$ & $4.5 \pm 0.2$ \\
\hline$P P$ & $132 \pm 13$ & $2.0 \pm 0.04$ & $131.5 \pm 0.3$ & $3.3 \pm 0.5$ \\
\hline$P S$ & $116 \pm 6$ & $2.1 \pm 0.1$ & $156.2 \pm 0.4$ & $1.9 \pm 0.3$ \\
\hline$P A$ & $130 \pm 6$ & $2 \pm 0.1$ & $171.6 \pm 0.9$ & $2.6 \pm 0.1$ \\
\hline$P E T$ & $223 \pm 8$ & $3.4 \pm 0.1$ & $251.2 \pm 0.2$ & $3.6 \pm 0.2$ \\
\hline
\end{tabular}




\section{Figure captions}

Figure 1. Evolution of storage modulus $E^{\prime}$ (a) and $\tan \delta$ (b) as a function of temperature for the different polymers. The left axis in Figure 1b applies to PMMA, PC, PP and PA while the right axis is for PS, ABS and PET.

Figure 2.Optical image of the residual nanoindentation imprints performed on: a) PC, and b) PMMA.

Figure 3.Representation of the harmonic contact stiffness versus the plastic deformation depth for PS.

Figure 4. Loading-partial unloading-reloading-fully unloading cycles for a PS sample after correction for the apparent tip defect following Loubet's method [14].

Figure 5. Mechanical properties obtained using the Loubet method, as a function of displacement into surface: a) Hardness, and b) Elastic modulus.

Figure 6. Comparison of mechanical properties obtained by Loubet and Oliver\&Pharr analysis: a) Hardness and b) Elastic modulus.

Figure 7. $\mathrm{E}_{\text {Loubet }} / \mathrm{E}_{\text {Oliver\&Pharr }}$ and $\mathrm{H}_{\text {Loubet }} / \mathrm{H}_{\text {Oliver\&Pharr }}$ as a function of the difference between the glass transition of each material obtained from DMA tests (see table 1) and the measurement temperature. 


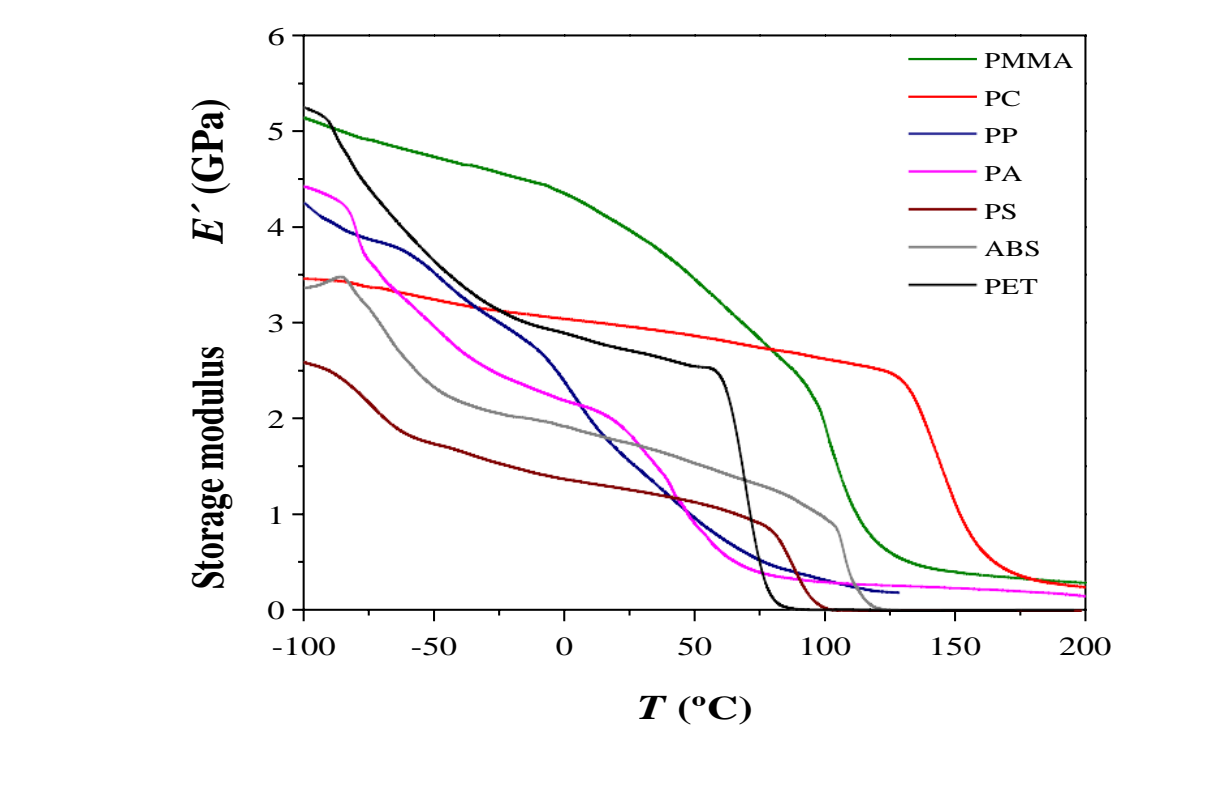

Fig. 1a

\author{
(20)
}

\title{
Figure 1a
}

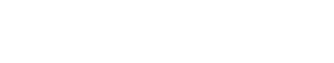

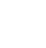

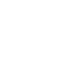
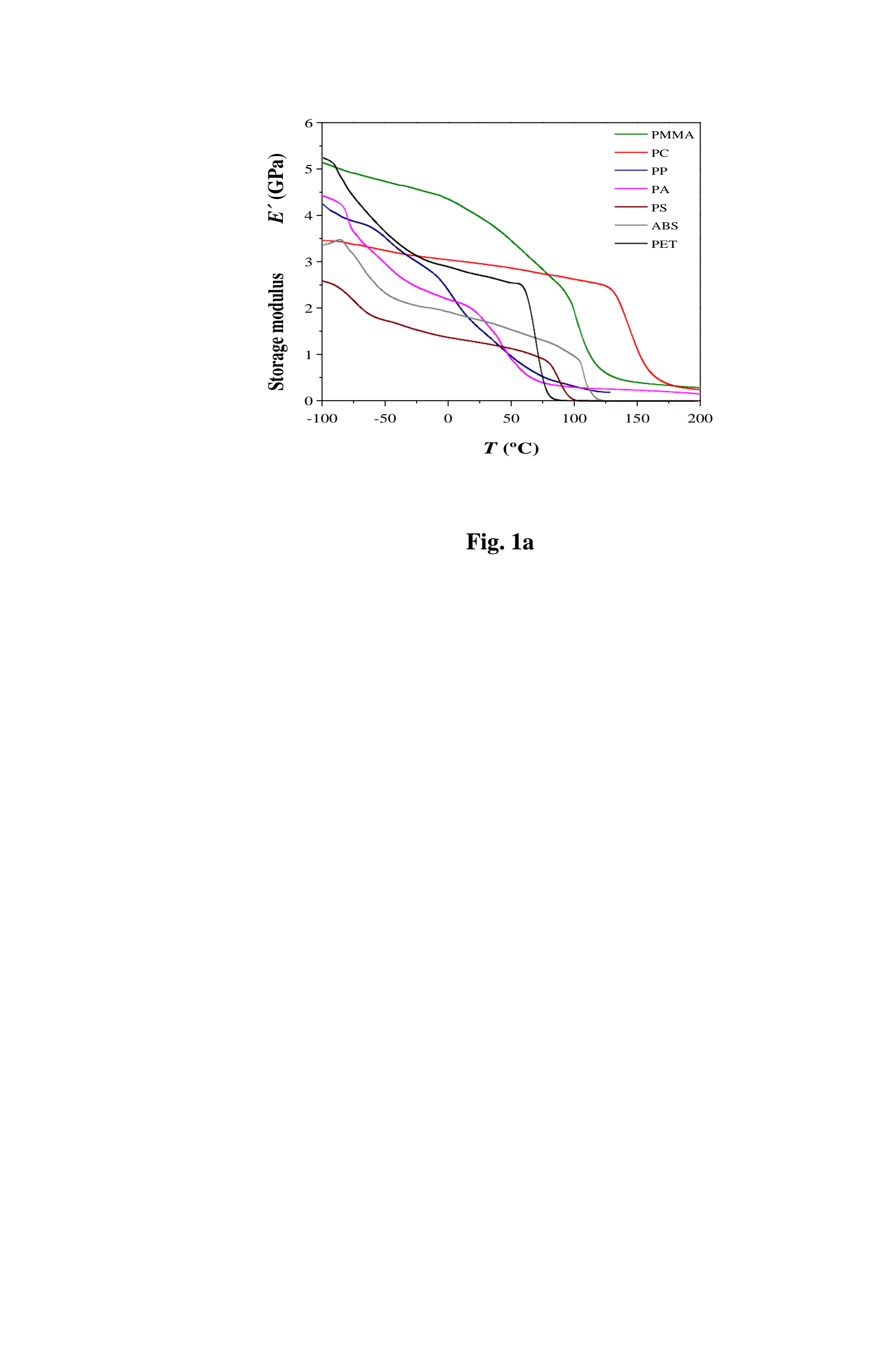


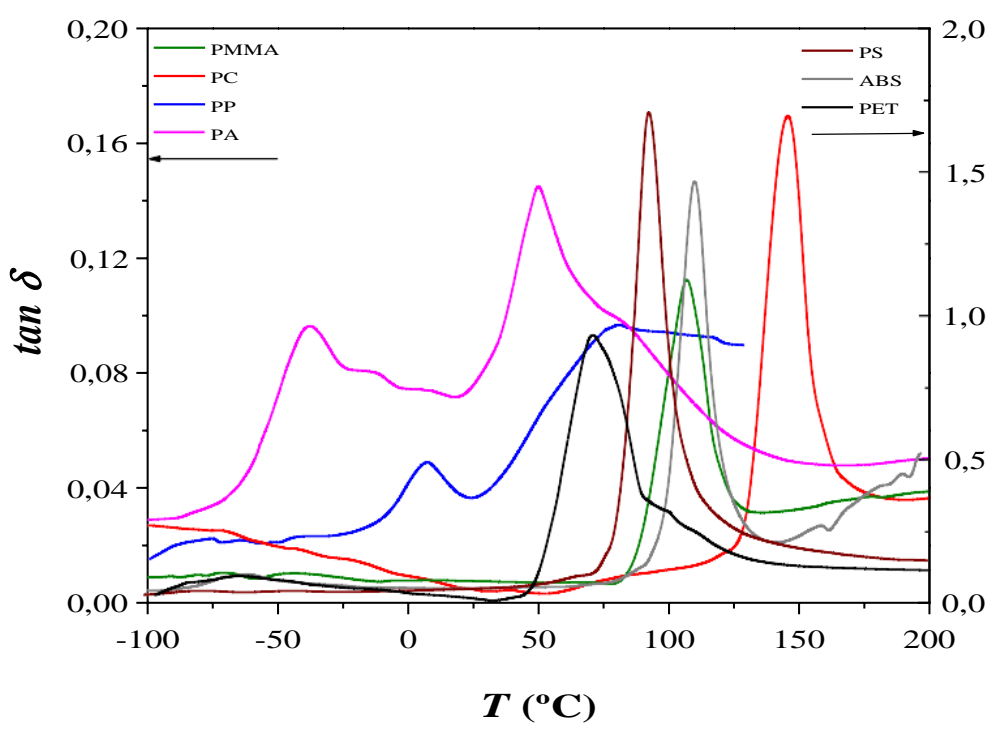

Fig. 1b 
Figure 3

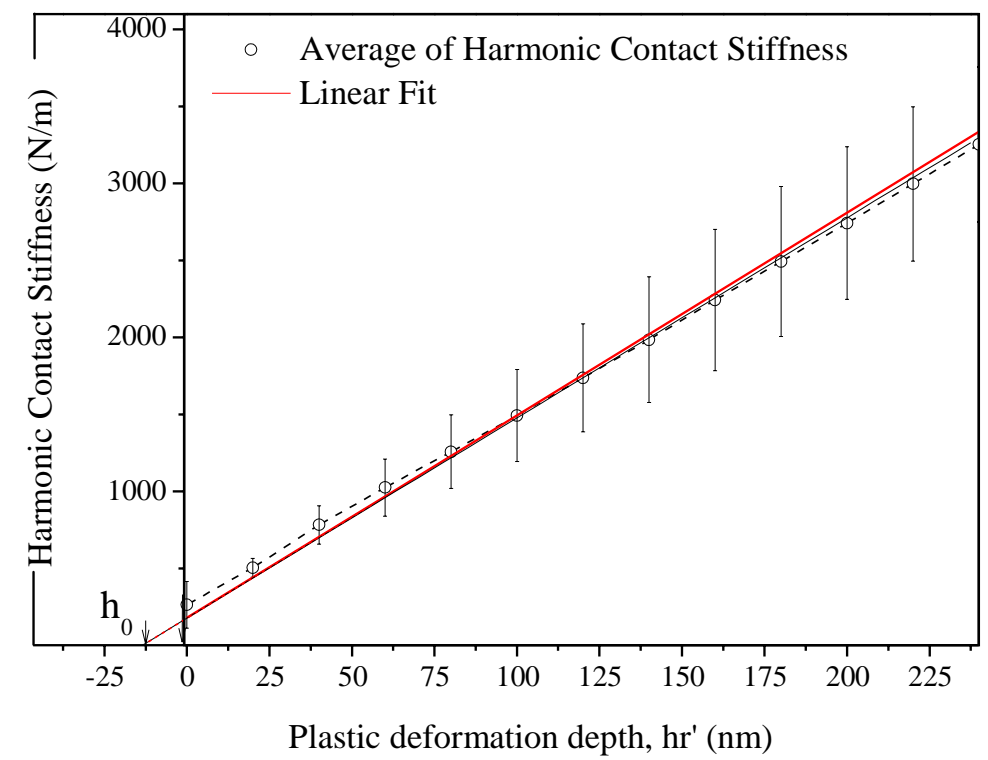

Figure 3 


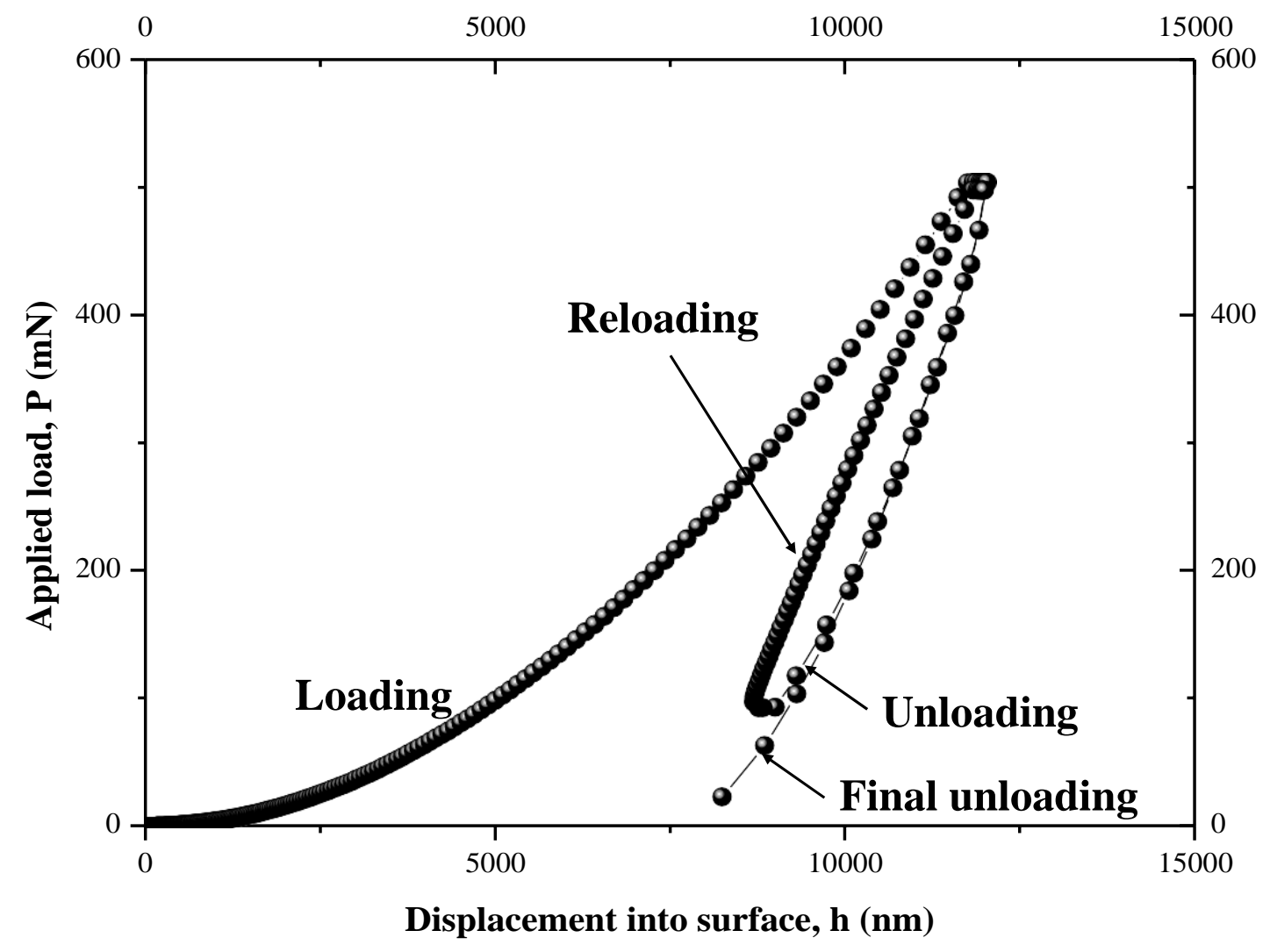

Figure 4 


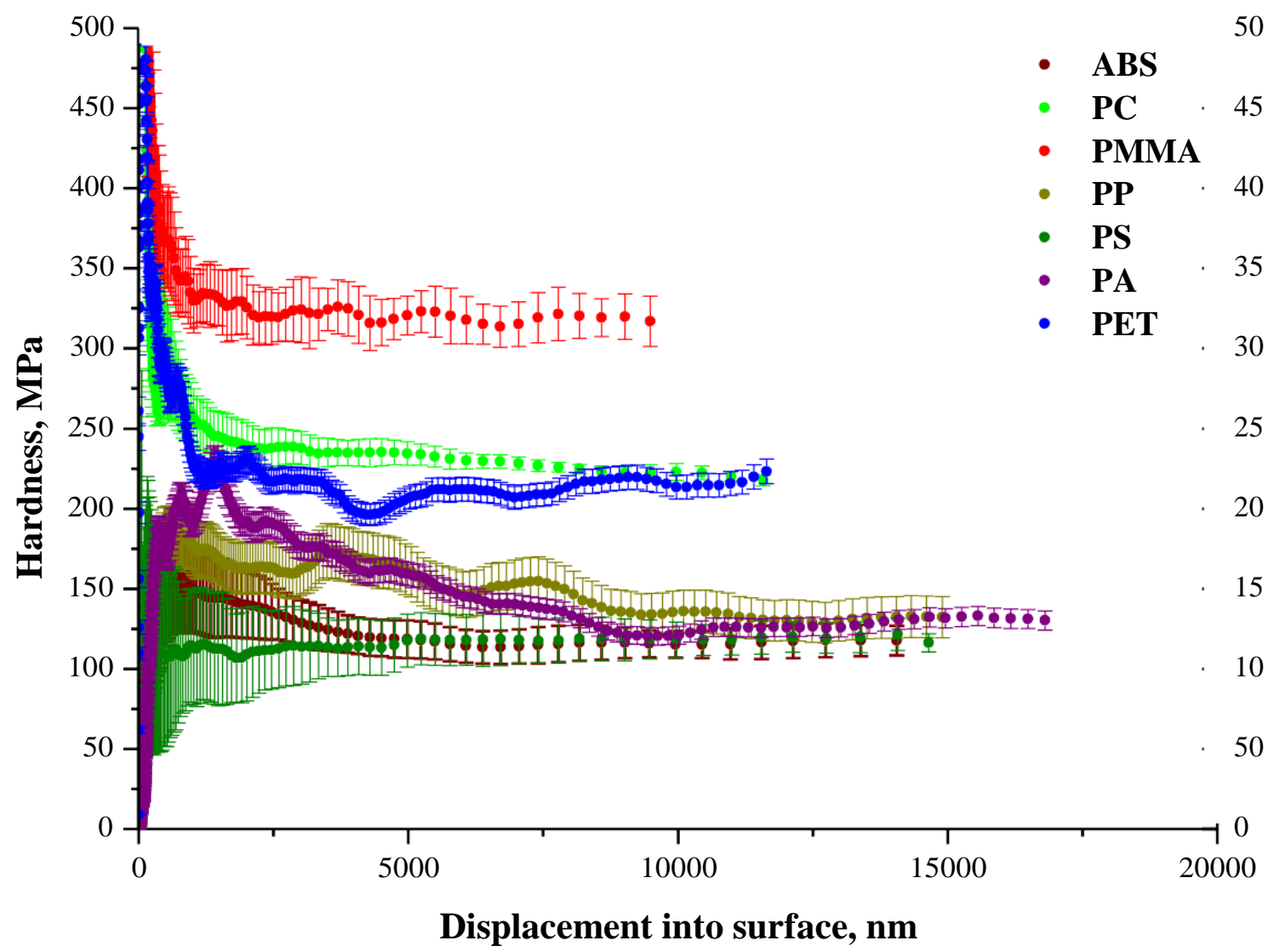

Figure 5a 


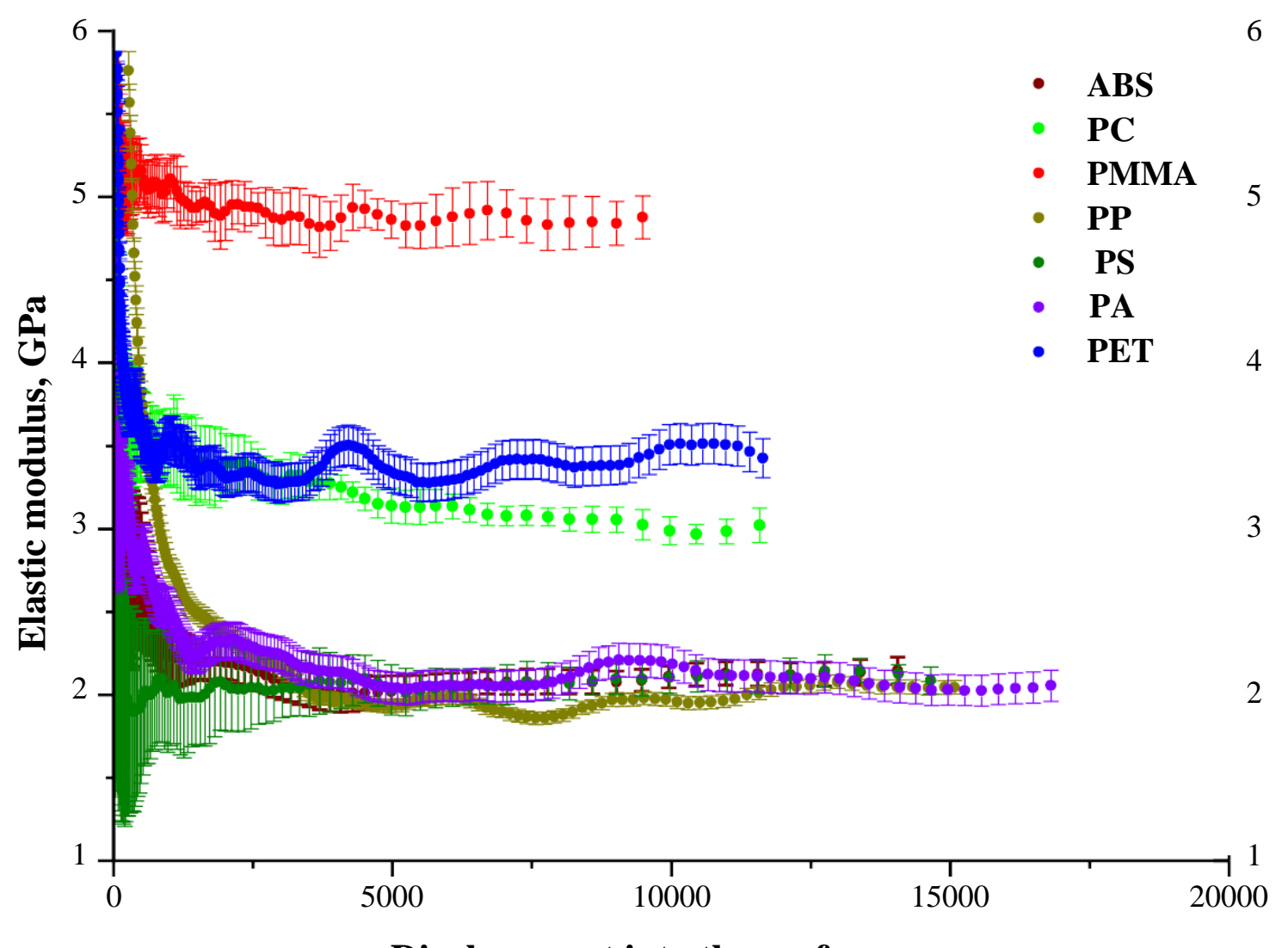

Displacement into the surface, $\mathrm{nm}$

Figure 5b 


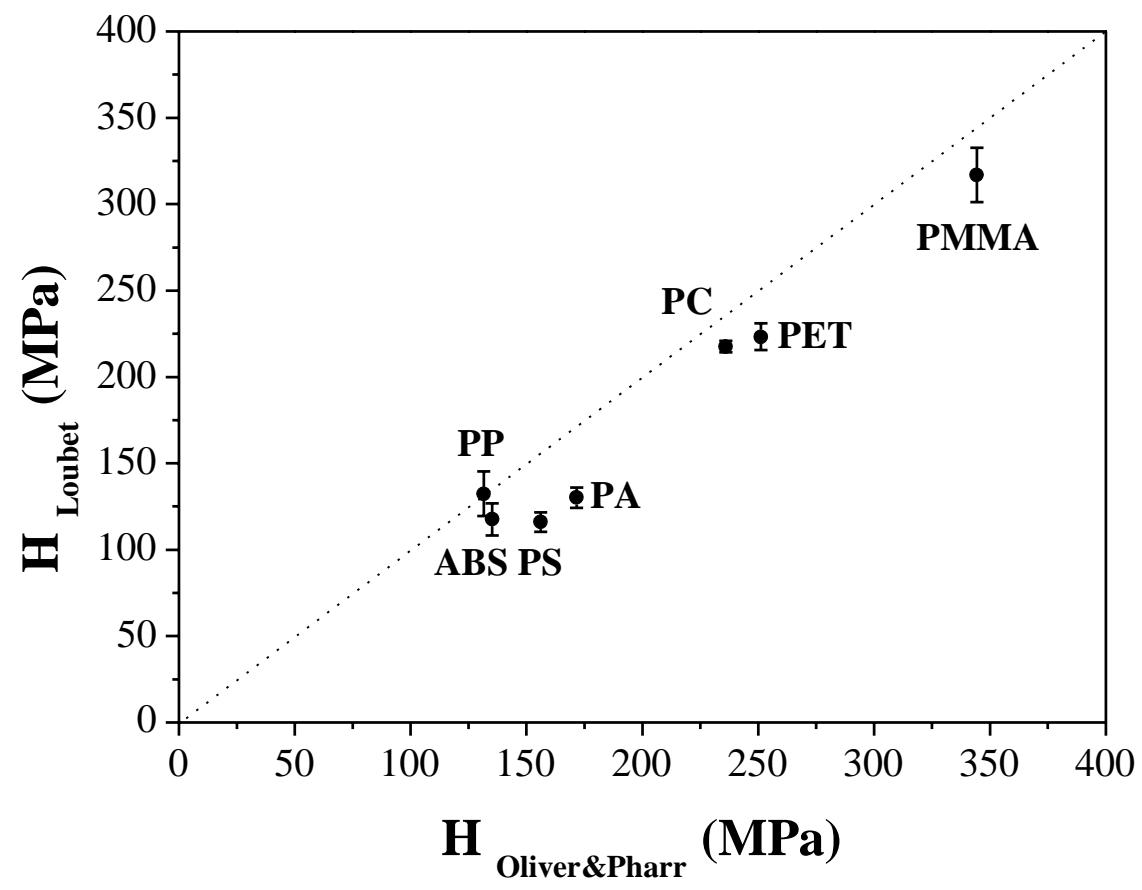

Figure 6a 


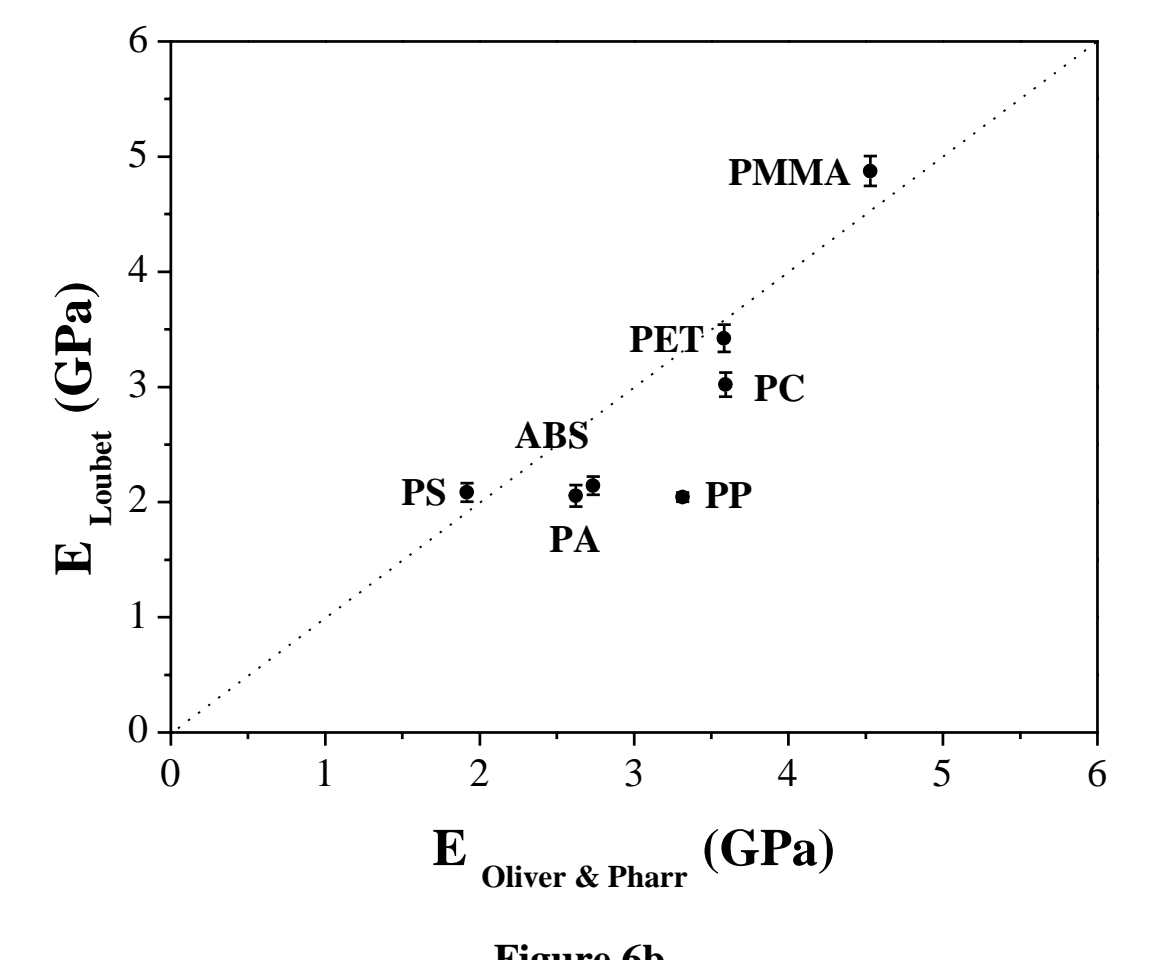

Figure 6b

Figure

(n)

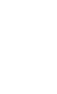


Figure 7
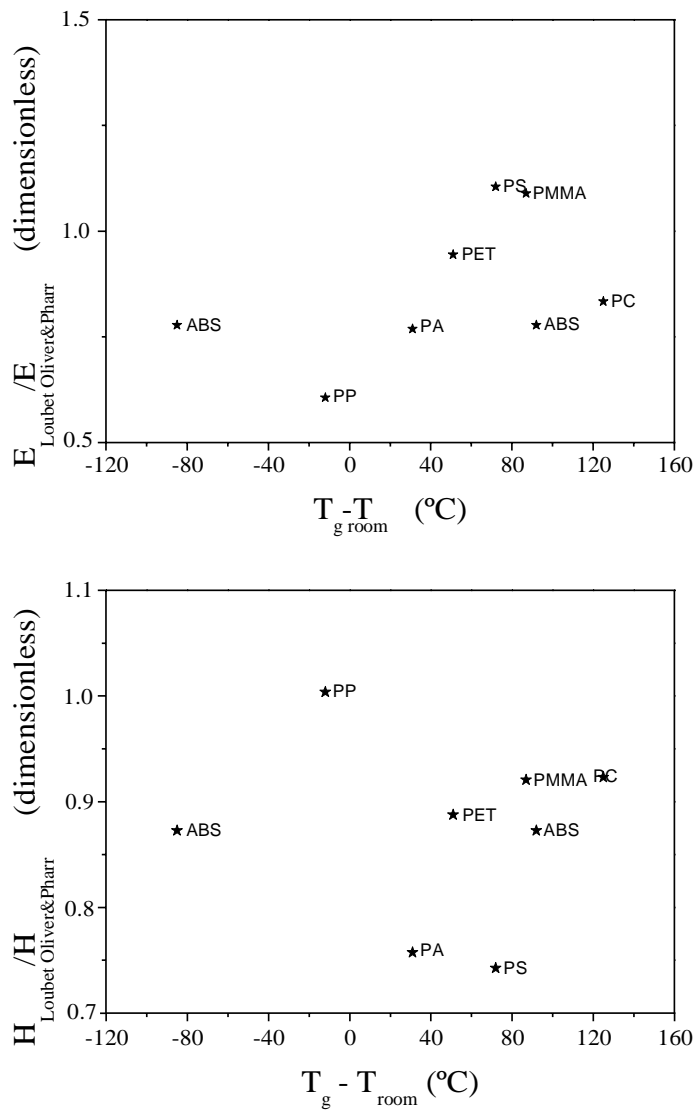

Figure 7 